Horizons philosophiques

\title{
À qui s'adressent les ouvrages de Herder? Notes sur Herder, sa conception du livre et du public
}

\section{Dominic Desroches}

Volume 13, numéro 2, printemps 2003

Herder (1744-1803) : le clair-obscur

URI : https://id.erudit.org/iderudit/801232ar

DOI : https://doi.org/10.7202/801232ar

Aller au sommaire du numéro

Éditeur(s)

Collège Édouard-Montpetit

ISSN

1181-9227 (imprimé)

1920-2954 (numérique)

Découvrir la revue

Citer cet article

Desroches, D. (2003). À qui s'adressent les ouvrages de Herder? Notes sur Herder, sa conception du livre et du public. Horizons philosophiques, 13(2), 1-22. https://doi.org/10.7202/801232ar d'utilisation que vous pouvez consulter en ligne. 


\title{
À QUI S'ADRESSENT LES OUVRAGES DE HERDER?
}

\author{
NOTES SUR HERDER, \\ SA CONCEPTION DU LIVRE \\ ET DU PUBLIC
}

Souvent, Herder a été compris, avec Humboldt, comme un philosophe idéaliste du langage, un précurseur de la linguistique. On l'a aussi reconnu comme un adversaire de Kant, dont il critiqua le tournant transcendantal. Grand défenseur de la culture et des contes populaires, Herder a été associé commodément au nationalisme allemand. Trop souvent, ajouterons-nous, les manuels de philosophie ont réduit Herder au rôle de Stürmer en pleine Aufklärung. En fait, Herder tente de répondre aux exigences de son siècle en repensant l'histoire à partir des limites des philosophes rationalistes, Voltaire et Kant, mais surtout à partir du langage. Or son projet philosophique, ont relevé les commentateurs, ne passe pas la rampe de la pensée critique et échoue sur les récifs de la poésie; ce constat, d'autant qu'il est partagé par nombre de spécialistes, appelle un problème précis : comprenons-nous bien l'œuvre de Herder? Les livres de Herder, si difficiles à lire et à situer, doivent-ils être lus dans l'orbe de Kant? Tel que le consignait Madame de Staël, arrivant à Weimar : "ses livres (Herder) semblent plutôt improvisés que composés ${ }^{1}$. Nul doute ici que Herder apparaisse à maints lecteurs comme un auteur qui manque de sérieux et dont l'œuvre, qui ne se saisit pas facilement, semble s'interpréter dans tous les sens. Ne cessant de reprendre ses textes et de les transformer, Herder paraît avoir tout publié sous le coup de l'émotion, dans le désordre. Mais est-ce le cas?

Dans ce texte, nous nous intéresserons aux rapports que Herder entretient avec le livre, les livres, et surtout ceux qui constituent son œuvre, en rappelant d'abord le contexte dans lequel Herder travaille, qui est celui de l'émergence de la "culture de l'imprimé». Nous cherchons à savoir ce que signifie pour lui écrire, écrire sur l'histoire à laquelle il appartient, écrire au moment où se construit un espace public en Allemagne, et donc écrire pour un public inséparable du peuple à venir. Ces questions sont essentielles : les textes sur 
l'histoire, sinon l'œuvre entière de Herder, ne pourront être compris que si l'on parvient à saisir la manière dont il rédige ses textes, ses livres, mais aussi et surtout à déterminer à qui il répond par l'écrit et à quel public (Publikum) il s'adresse. Écrit-il des livres ou formule-t-il les balises d'un «mouvement" à venir? À qui veut-il parler d'histoire des peuples? Veut-il participer à une culture nationale émergente? $\mathrm{Ne}$ cherche-t-il pas simplement à produire un effet sur ses lecteurs? Bref : qu'entend Herder par «livre», par «œuvre»?

\section{Écrire après le génial Hamann : du fragment au livre}

Pour mieux cerner notre question, rappelons que ses premiers pas en philosophie, Herder les fait dans les cours de Kant. Si Kant est un excellent professeur, Herder s'avère original, car il transforme déjà le contenu des cours en le versifiant. Celui qui avait quitté Mohrungen en 1762 pour aller à Köningsberg rencontre Hamann la même année, à la librairie de Kanter, que fréquentait Kant. Cette rencontre, dans une librairie, fut décisive pour sa conception du livre, de l'écriture et pour l'histoire de la littérature allemande. De 14 ans son aîné et auteur connu - il avait publié ses Mémorables socratiques et Esthetica in Nuce -, Hamann laisse une impression profonde sur l'assistant d'études du Collegium Fredericum. Dès cet instant, Herder se retrouve entre deux géants : Hamann et Kant. Autant enviable que difficile, cette position de trait d'union perdurera et le marquera. La place qu'occupe Herder est si délicate qu'elle pourrait servir de première et de dernière explication à la future querelle avec Kant, en 1785. Mais en 1762, cela n'existe pas. Herder, formé à l'éducation piétiste et à la lecture de la Bible, accueille bien les idées véhiculées par Hamann, "le Mage du Nord", qui vont donner naissance, au tournant des années 1770, au Sturm und Drang- ou Geniezeit- un courant littéraire très important.

C'est bien sous l'influence de Hamann que Herder commence à écrire dans un style réactionnaire au rationalisme des Lumières français. Hamann, qui attend son public, se fait connaître en tirant de faits divers des "occasions" de rédiger des textes ironiques et prophétiques visant à créer des effets précis sur le lecteur, souvent pour rendre, à la façon piétiste, une Stimmung des Écritures. Hamann avance masqué et communique toujours indirectement. Son public reste, en 1762 en Prusse, incertain2. Or après avoir expliqué à Kant pourquoi à ses yeux le projet de l'Encyclopédie a échoué, Hamann (qui se prétendra le génie de Kant si Kant se présente comme le 
Socrate de Königsberg...) se voit investi de la tâche de conseiller Kant sur un livre à venir. Dans sa lettre, il lui écrit, au sujet de sa vision du livre, ces indications spirituelles : "L'esprit de notre livre doit être moral. Si nous ne le sommes pas nous-mêmes, comment pourrons-nous communiquer cet esprit à notre ouvrage et à nos lecteurs?3" C'est que notre rapport au monde dépend, explique-t-il, de notre rapport à Dieu et les questions concernant Dieu et la création ont leur racine dans la parole. Ainsi Hamann peut-il alors affirmer que le monde est langage et que nous écrivons de la manière dont nous nous comprenons nous-mêmes. Cela lui permet de mettre à jour une interprétation originale de la nature, comme l'illustre la suite de notre lettre datant de 1759, l'année de la publication de ses Mémorables socratiques:

La Nature est un livre, une lettre, une fable (au sens philosophique) ou comme vous devez l'appeler. À supposer que nous en connaissions toutes les lettres aussi bien que possible, que nous sachions même la langue dans laquelle ce livre est écrit, tout cela est-il suffisant pour comprendre un livre, pour en juger, pour en obtenir un caractère ou un extrait? II faut donc plus que la physique pour expliquer la Nature. La physique ne donne que l'A.B.C. La Nature est une équation d'une grandeur inconnue, un mot hébraïque dont seules les consonnes sont écrites, et auquel l'entendement doit ajouter les points-voyelles.

Nous n'écrivons pas seulement pour une nation, comme les Encyclopédistes français, mais bien pour un peuple qui exige des peintures et des poètes : mediocribus esse poetis non homines, non dii, non concesse columnaet.

Prenant le contre-pied de Galilée pour qui la nature est un livre ouvert, le luthérien Hamann estime plutôt que la nature est un livre scellé, un signe caché, face à l'inventivité des tentatives scientifiques. D'après lui, la nature et l'histoire constituent les «deux grands commentaires de la parole de Dieu». Tout est langage : la création est un livre scellé dont la clé est la foi (Glaube), une parole (Wort) dont les signes ne coïncident pas avec le réel. La nature est une énigme qui ne se laisse comprendre que par la coïncidence des opposés, l'absurde face à la raison, s'il faut en croire une lettre à Jacobi de décembre 1784 :

Dieu, la nature et la raison entretiennent des relations aussi 
intimes que la lumière, l'œil et tout ce que celle-là révèle à celui-ci; ou encore que le centre, le rayon, la périphérie d'un cercle donné; ou encore que l'auteur, le livre et le lecteur. Mais où se trouve l'énigme du livre? Dans son langage ou dans son contenu? Dans le plan de l'auteur ou l'esprit de l'interprète?5

La conception du livre de Hamann repose sur une pensée du langage, elle-même tributaire d'une esthétique "symboliste" pour laquelle le langage est formé à partir des images provenant des sens et la réalité ne peut, à nous les hommes, "nous parler qu'en similitudes", car "toute notre connaissance est sensible et figurée»6. Les concepts sont froids et éloignés des premières images, alors que le signe appelle l'esprit et inversement. C'est ainsi que le réel mérite une interprétation en tant qu'art, les images et les analogies étant la raison même des choses. Ces dernières se donnent à nous dans une poésie aussi belle et harmonieuse que la création elle-même. Dans Festhetica in Nuce, Hamann nous avait révélé que «la poésie est la langue maternelle (Muttersprache) du genre humain", ce que retiendra Herder, boudant les concepts froids, en rédigeant tous ses textes. Pareille esthétique ne saura, cependant, être vraiment indépendante d'une herméneutique biblique pour laquelle l'homme, devenu interprète, doit déchiffrer les signes divins dans la nature et chercher à faire parler le "grand livre de Dieu».

Or revenons à Herder sur qui porte la majeure partie de nos efforts. Les livres de Herder s'inspirent de ces idées. En effet, Herder transpose l'esthétique de Hamann dans sa démarche littéraire et philosophique. Son écriture conjugue les réminiscences bibliques aux métaphores et aux analogies. Ce qui est valorisé par Herder, c'est l'aphorisme, la réplique ironique, le génie, mais peu le lecteur. Or le style d'écriture de Herder vise néanmoins (un écrivain peut-il se détacher, dit Hamann, de ses lecteurs?) à produire un certain effet (Wirkung) sur les lecteurs, ceux à venir, afin de construire un public. Herder sentira le besoin de réunir ce public sans structure. Notons que pour cette tâche, sans trop anticiper, Herder opte pour un style qui privilégie la supériorité de la vitalité, l'inspiration et la spontanéité (pensons au nombre de points d'exclamation dans ses textes) plutôt que le mûrissement des idées . Stürmer et écrivain public incertain, il ne cessera de caresser le projet de construire, dans le sillage de Lessing et d'Abbt, un public, forgeant par là notre catégorie d' “écrivain public», notamment en contestant les Lumières et en 
voulant réunir, dans une Allemagne encore médiévale, les grands groupes sociaux (les nobles, la classe moyenne et les autres), euxmêmes divisés en groupuscules ${ }^{8}$. Morale de ceci : le style ne peut se détacher des buts poursuivis par celui qui écrit.

Cela dit, l'élaboration de l'œuvre de Herder suit un fil conducteur qu'il faut savoir reconnaître : l'étude du langage, qu'il dit poétique, le conduit à formuler une philosophie de l'histoire. À Riga, Herder travaille comme pasteur. Quand il est en chaire, sa mission est de donner la Parole. Or Herder commence à préciser son champ de réflexion et songe aux liens qui unissent la langue et la culture, la culture aux peuples. À ce moment, entre 1765 et 1766, il revoit Hamann à Mitau. Loin d'être le fruit du hasard, cette rencontre est stratégique puisqu'il compte sur Hamann pour agir comme conseiller dans la préparation de son œuvre. Après Kant, Hamann doit conseiller Herder sur ce qu'est un livre. Le rôle du Mage peut alors être bien défini : faire des "Fragments" Sur la nouvelle littérature allemande un livre. En effet, le Mage, dont le génie est reconnu et la notoriété n'est plus à faire, doit l'aider à passer des esquisses à l'œuvre, des écrits presque toujours anonymes à l'œuvre signée. Mais comment expliquer ce voyage jusqu'à Mitau, dans le froid et sur la glace, que Herder fait au péril de sa vie, si ce n'est pas pour apprendre à écrire un livre?

Pourtant, les Fragments de Herder publiés sans nom d'auteur en 1767 (comme les Sylves critiques deux années plus tard) furent bien accueillis par la critique, y compris par Kant ${ }^{9}$. Herder n'a pas de raisons valables de craindre le public, bien que certains critiques n'aient pas manqué de relever l'«unité artificielle» des Fragments, "fragments de fragments" ou vus comme un livre en devenir... Nicolaï, l'Aufklärer berlinois, déplorait bien un ordre caché qui n'était pas visé au départ, mais sans plus. Pour ses lecteurs, Herder semble ambivalent, paraît toujours improviser et sa réputation, il le sait, le suit comme une ombre. Après le dévoilement de son anonymat, il quitte curieusement Riga...

Le rapport difficile qu'entretient Herder à l'écriture transparaît donc dans l'œuvre, car Herder, qui est pressé de faire "sonner" les mots aussi bien que Hamann, montre d'énormes difficultés à supporter la distance entre l'écrit, le texte, et sa diffusion, le livre. Pourquoi? Pourquoi Herder est-il insatisfait du résultat? Ses modèles sont-ils trop grands? Incapable de terminer un livre, il propose, comme le symptôme d'une maladie, des projets : les Fragments, qui ne sont pas 
un livre (il dira plus tard qu'ils forment "un recueil d'écrits mêlés") constituent un programme. C'est ainsi que Herder commence une œuvre dont la méthode n'est pas celle de résumer des pensées, mais de les croiser et de les superposer, une œuvre qui ne sera jamais linéaire et ne fera pas l'unanimité, comme il le pressentait lui-même dans ses Fragments. Ces derniers, qui sauront plaire à Schlegel, Arnim et Uhland, portent sur la compréhension d'une œuvre littéraire et peuvent être interprétés comme l'essai inachevé d'un système général de la langue.

Toujours est-il que Herder décide à nouveau de se déplacer. Durant son voyage en France, il rédige beaucoup. En voyage, il adopte le journal comme forme littéraire. II y note ses impressions quotidiennes, journal "écrit non pas pour le public, mais pour luimême». Sans doute parce qu'il a pris une trop grande liberté dans le propos et le style, il ne publiera pas Le journal de mon voyage en 1769. En France, il se retrouve dans une culture littéraire et livresque très différente de celle qu'il a connue en Prusse. Comme l'illustre sa critique de la culture française (élève ici de Lessing), il refuse ce monde livresque et de salons. Un seul exemple suffit: Herder ne partage pas, comme Kant, le projet de livres des encyclopédistes Diderot et D'Alembert. Selon lui, cet encyclopédie n'est pas un triomphe, mais le signe de la décadence de la pensée française, qui est de plus en plus «abstraite» (sic). L'encyclopédie rejoindra un public cultivé, bourgeois pour reprendre un des mots préférés d'Habermas, mais pas le public auquel pense Herder, celui qui accomplit l'histoire. En cela, son expérience contient en germe la vision de l'Auklärung qu'il opposera à Kant10. Les Français, précise-t-il dans son journal, écrivent des résumés et des abrégés (ce à quoi ressemblaient assez ses Fragments de $1767 \ldots$..), ils n'ont plus rien à écrire - au sens de force vitale - . Durant ce voyage, il ne publiera rien. Les choses changeront toutefois avec la remise du premier prix à son Traité sur l'origine des langues. Herder, qui venait de soutenir avec succès la thèse d'une origine humaine au langage contre son ami Hamann, reprend confiance dans l'écrit et se lance dans la rapide rédaction de Auch eine Philosophie der Geschichte (Une autre philosophie de l'histoire). Ce livre au titre «ironique et satirique»11 peut nous apprendre beaucoup sur la façon dont le jeune Herder envisage l'écriture, le livre et sa vocation de philosophe. 


\section{Le grand livre de Dieu dans Auch eine Philosophie (1774)}

Après 1770, Herder se penche plus sérieusement sur l'histoire : la langue humaine n'a de "sens" que dans la culture et l'histoire. Dès 1771 en fait, il se retrouve dans un petit appartement de Bückeburg, bien décidé à reprendre sa production. Cependant, il ressent l'isolement. Plus tard d'ailleurs, Herder retravaillera les textes rédigés durant cette période de solitude (Le Plus Vieux Document du genre humain, les Pages provinciales, les Éclaircissements pour le Nouveau Testament), période durant laquelle il ne s'intéresse qu'à la Bible, livre dont l'esprit est hautement moral, "livre des livres" qu'il interprète à l'aide des intuitions et des directives herméneutiques de Hamann.

Mais la crise existentielle devra durer deux années encore. En juillet 1773 , Herder se plaint à nouveau à Hamann. Raison : sa production littéraire est arrêtée. Nous pouvons affirmer ici que cela tient, du moins en partie, au rapport équivoque à Hamann : tantôt il fait figure de disciple en écrivant, sur sa recommandation, des Fragments portant sur l'histoire du langage et de la poésie, tantôt il critique la théorie hamannienne de l'origine divine du langage dans son Traité sur l'origine des langues, tantôt enfin il utilise son herméneutique pour déchiffrer et interpréter la Bible. L'influence de Hamann, cependant, ne pourra être plus nette que dans la dernière partie de Auch eine Philosophie. Mais avant de voir comment l'auteur construit sa philosophie de l'histoire, il convient de dire un mot sur le contexte et la signification de ce livre, si important aux yeux de Herder.

Rédigé en trois semaines seulement à l'été de 1773, peu de temps en fait après De la manière et de l'art allemand, Auch eine Philosophie est un ouvrage inspiré, mais non signé. Herder avait pris soin de faire copier son livre par une main étrangère afin qu'il conserve son anonymat, comme il l'avait fait pour les Fragments. Voulant miner de l'intérieur l'orgueil des Lumières naissantes (Herder voudra, lui aussi, expliquer le progrès, la liberté et créer un espace public), l'ouvrage s'annonce sur un ton polémique, de manière à en forcer la lecture. Dans un style plus prophétique que didactique, Herder pose le problème d'une "philosophie de l'histoire" en un sens tout différent de Voltaire et des rationalistes. En effet, il ne suffit pas, estime Herder, de reconnaître simplement le progrès et de chercher à l'expliquer par la raison, telle une mécanique, encore faut-il réconcilier la progression de l'histoire avec la Providence. Coincé entre l'idée d'un 
progrès continu de l'histoire (le fameux Fortgang) et la défense de la volonté de Dieu, Herder parvient néanmoins à esquisser une philosophie de l'histoire au centre de laquelle pourra se développer une conscience historique nouvelle pour la modernité, issue du christianisme, comme l'a fait remarquer Gadamer'12, une pensée riche dans son ouverture, riche justement parce qu'elle prend acte de l'altérité propre à la notion de culture. L'assise luthérienne est toutefois si forte qu'il ira même jusqu'à affirmer que l'homme, il faudra se le rappeler, n'est que "le but ou un simple instrument" (Werkzeug) du destin ${ }^{13}$.

Considérant la Bible comme le livre «le plus ancien", il cherchera à la réhabiliter contre Voltaire, car le langage est le mouvement de l'histoire. Herder reste donc persuadé qu'il existe une antériorité du langage poétique sur la raison. Sauf que dans ce contexte, écrire sur l'histoire est périlleux parce que cela suppose que l'homme, simple Werkzeug, reconnaisse que "le genre humain fait partie du grand plan d'ensemble divin qu'une créature isolée ne peut embrasser du regard" 14. L'horizon de l'histoire est beaucoup trop large pour la vue de l'historien ou du philosophe ${ }^{16}$. L'illusion que Herder tient à dénoncer est sans contredit celle des Lumières qui se croient le centre du monde et de l'histoire. Illusion, car dans le plan divin, le centre est partout! À l'aide d'analogies organicistes, il précisera sa critique : "Sous le grand arbre du Père de tout chose (...) quelle petite fibre du feuillage de cet arbre je puis bien être! Une petite virgule, un petit tiret dans le livre des mondes»16. À la manière apocalyptique hamannienne, Herder ajoute : «Philosophe, veux-tu honorer et utiliser la situation de ton siècle : le livre du passé est étalé devant toi! fermé de sept sceaux; livre merveilleux plein de prophéties : la fin des jours est venue jusqu'à toi, lis!17». Celui qui sait déchiffrer l'énigme, traduire le Livre, verra dans le passé l'avenir. C'est donc le grand livre de Dieu qu'il faut savoir traduire pour découvrir le sens de l'histoire. La tâche du philosophe, qui n'échangera jamais l'éternité pour l'historicité, mais les fera travailler de concert, relève d'une herméneutique de la poésie, puisqu'elle consiste à retrouver les signes divins dans la nature et à s'ouvrir au passé (dans l'acte même d'écrire) pour prédire l'avenir : "Ce que tu écris devrait en chaque mot être digne du monde entier et de l'éternité parce que toi (du moins pour les matériaux et les possibilités), tu écris pour le monde entier et l'éternité». Certes, l'ensemble de l'histoire est grand, le Livre du créateur est long à déchiffrer, l'homme n'ayant devant lui que des détails : 
mais où dans chaque détail se révéla toujours une telle unité indéterminée et orientée uniquement vers l'ensemble! Où de petites connexions offrent déjà une si grande signification, et où pourtant les siècles ne représentent que des syllabes, les nations que des lettres, peut-être des signes de ponctuation qui ne signifient rien par eux-mêmes mais dont l'importance est si grande pour faciliter l'intelligence de l'ensemble !18

Notre interprétation se voit à nouveau confirmée plus loin lorsque Herder ajoute :

Cela précisément me garantit que moi je ne suis rien, mais que l'ensemble est le tout! Quelle œuvre (Werk) (...) Qui suisje pour juger, moi qui ne fais que traverser en biais la vaste salle et lorgner un coin du grand tombeau recouvert où une lumière vacillante laisse plus d'obscurité? (...) Qu'aurais-je à dire du grand livre de Dieu (grossen Buches Gottes) qui s'étend sur les mondes et le temps! dont je suis à peine une lettre et n'aperçois à peine trois lettres autour de moi'19?

Ce passage illustre à merveille que Herder prend au pied de la lettre l'enseignement de Hamann et que sa conception du livre, en 1774, est divine. L'écrivain, une sorte d'herméneute mais de l'avenir, se limitera à décrire et à interpréter ce qui se passe sous ses yeux à la faveur d'un art poétique inspiré - Dieu seul pouvant "voir» le plan d'ensemble - dérouler le fil, dont émanent les signes, de ce progrès continu (Fortgang).

\section{Écrire un livre sur l'histoire : \\ raison, horizon et interprétations}

Si Herder se bat sur plusieurs fronts, sa critique principale porte sur la conception de l'histoire des Lumières. Instrument du grand livre de Dieu, le philosophe, qui participe au projet des Lumières, cherchera à comprendre, à même la description qu'il en donne, l'originalité de chaque époque : le point et la virgule, aussi différents soientils, ponctuent le même texte. Autrement dit, Herder veut saisir chaque nation à même sa formation (Bildung), en devenir. Plus qu'un livre d'histoire, Auch eine Philosophie critique l'Aufklärung qui rapporte tout à la mesure de sa propre époque. Se gardant des excès rationalistes, Herder veut comprendre chaque époque à partir d'elle-même. L'originalité herdérienne est assurément de vouloir lire l'histoire 
comme un "horizon" dynamique des cultures et de pointer l'erreur des philosophes qui interprètent toujours l'histoire comme un progrès sans ruines ni pertes.

Mais de la perspective de Herder donc, qui accorde à l'œuvre de Dieu le seul titre de «livre», comment signer un livre d'histoire avec une introduction, un développement et une conclusion? En régime luthérien strict, comment, tenant compte de la Bible et la nature qui émane d'elle, écrire un livre définitif sur l'histoire? Comment écrire un livre, qui suppose toujours un écrivain extérieur à ce qu'il décrit, sans répéter les erreurs de Voltaire et d'autres, qui unifiaient abstraitement les grandes périodes de l'histoire et, par là, oubliaient les forces à l'œuvre et leur propre mouvement d'écrivain? Car si toute époque est incommensurable aux autres, celle à partir de laquelle l'histoire s'écrit l'est aussi.

Certes, Herder est l'un des premiers penseurs à dire que le centre de l'histoire est partout20. II se montre ainsi conscient du mouvement réalisant l'histoire, de l'ouverture à la force et au sens, c'est-à-dire que le récit historique dépend de celui qui l'écrit. II n'est alors pas étonnant qu'avec Herder, nous ayons affaire avec un écrivain qui écrit beaucoup et dont la vie aura été d'écrire et de se reprendre, de se dédire et de réaffirmer autrement, tentant de préciser toujours plus ce qui est "sens". Ainsi dirons-nous que par ses textes, il se présente comme un écrivain (parmi d'autres) réfléchissant sur l'histoire - ce sont précisément les peuples qui constituent les acteurs de l'histoire - et réinterprétant celle-ci à laquelle il ne cesse jamais d'appartenir et de participer. L'histoire est toujours "sens".

Ces mots nous conduisent à Gadamer, qui a très tôt écrit sur l'œuvre de Herder'21. Gadamer a reconnu en Herder un penseur de la conscience historique. Herméneute, il a vu que l'effectivité historique n'est rien d'autre que l'efficacité de la force et que Herder, attentif au concept de "forces organiques" (Organischen Kräfte) a développé une forme de vie propre à l'expérience donnée, détachée des livres ${ }^{22}$. Comme l'a fait voir Gadamer, qui a certes tort d'aseptiser parfois Herder de l'Aufklärung, le "testament philosophique et historique» de Herder se trouve dans la «finalité interne de la nature de l'histoire23». En d'autres termes, tout est sens et force. Mais le coup de maître de Gadamer est sans doute d'avoir compris que l'idée d'horizon, empruntée à Herder ${ }^{24}$ - l'horizon est le champ de vision qui comprend et inclut tout ce que l'on peut voir d'un point précis 25 - est un concept 
clé de notre rapport au sens et d'en avoir fait un concept déterminant de son herméneutique. Ces précisions sur la conscience historique nous permettent d'éclairer l'écriture de Herder. Parce que la compréhension humaine procède d'un horizon de sens et d'une histoire, Gadamer fait glisser l'horizon au sens géographique vers l'horizon au sens historique et herméneutique, c'est-à-dire comme fenêtre pour l'interprétation. Quand il propose cette base à son herméneutique, Gadamer fait avancer une intuition centrale de Herder (qui s'oppose aux Lumières figées) lorsqu'il écrit, dans son opus magnum :

L'homme sans horizon est celui qui ne voit pas assez loin et qui, pour cette raison, surestime ce qui est proche de lui (...) Quiconque ne manque pas d'horizon sait apprécier à sa juste valeur la signification de toute chose comprise dans cet horizon, en ce qui concerne sa proximité et son éloignement, sa grandeur et sa petitesse. De même, élaborer la situation herméneutique est-ce acquérir l'horizon d'interrogation approprié aux questions qui se posent à nous sur la tradition26.

Herder refusait de voir le problème de distance entre les faits objectifs (ceux de la tradition) et l'écrivain. Pour lui, l'écrivain devait s'approprier immédiatement le sens, même s'il se savait fini face au plan de la Providence. Ainsi Herder ne réalisait pas qu'il était trop proche de l'action qu'il décrivait - selon sa méthode -; il n'a jamais, et c'est une faute grave, le temps de fouiller les sources historiques. Toujours inachevés, ses textes sur l'histoire doivent s'interpréter dans cette direction, c'est-à-dire, en théorie esthétique, comme faisant partie d'une œuvre "ouverte" au sens où elle assure le multiple et la différence. Incapable d'assumer l'achèvement, Herder ne propose pas de livres mais une œuvre ouverte au sens moderne : attentive aux perspectives historiques, elle est mobile dans sa structure et virtuelle en sa genèse. Cela lui permet d'engendrer une multitude de significations à l'intérieur même de sa formation; les pires lectures sont ainsi possibles. Bref : l'œuvre est ouverte parce qu'elle refuse de geler la forme et le sens. Plus rigoureux quant à la distance et ses effets, Gadamer aura compris que la tradition porte le sens et que nous répondons aux questions que notre langage nous permet de poser.

Mais Gadamer n'est pas Herder. Celui-ci demeure incompris. Et c'est en grande partie le drame de Herder que de s'adresser, de manière ambivalente, à un public dont les intérêts semblent figés 
dans une époque, un moment de l'histoire. Si l'Aufklärung réalise qu'elle accomplit un pas, qu'elle incarne un progrès par rapport aux siècles antérieurs, elle en est fière et fait tout ce qu'elle peut pour arrêter le temps, le nommer, en quelque sorte "immortaliser" son époque. Herder ne se reconnaît pas dans ce Zeitgeist et devra composer avec cette réalité, notamment en explorant des formes de communication.

Voilà peut-être pourquoi il écrit si souvent de manière anonyme, pour ainsi dire inconnu et inconnaissable, à l'ombre de Kant et de Goethe. Durant ces années de production, il se lève très tôt pour concilier lecture, travail et écriture27: il écrit à haute vitesse (une écriture "automatique" avant l'heure), presque trop, sans distance, tout en soutenant sa correspondance. La place du livre est ici auxiliaire : elle se situe, comme l'a vu Pénisson, entre la lettre privée, le sermon et une publicité difficile à assumer. Celui-ci a selon nous raison d'écrire que "ses rétractations et reprises, qui sont des éléments constitutifs de sa production, témoignent d'un auteur s'imaginant avoir encore possession et maîtrise de ce qui est publié, comme d'une parole qu'on pourrait retirer ou d'une correspondance que l'on pourrait modifier suivant la réaction du destinataire ${ }^{28}$ ». Tout se passe un peu comme si l'écrivain Herder voulait suivre lui-même les mouvements de l'histoire en son écriture, en pleine effectivité, une écriture qui agit, qui produit un effet et qui se modifie au fil de ce qu'elle décrit. En ce sens, l'écriture est toujours action et Pénisson a derechef raison de rattacher le travail d'écriture de Herder au réseau de significations de Würkung, würken, sinon à la Wirklichkeit (effectivité) elle-même . Voilà pourquoi nous avons tant insisté pour rapprocher Herder de Gadamer. Mais ce constat, qui marque les limites de tout livre sur I'histoire et de toute philosophie de l'histoire, ne peut que nous mener sur le chemin de la querelle avec Kant, en 1785. Mieux : il nous aidera à la comprendre.

\section{Le livre de l'histoire inachevé :}

\section{la recension des Ideen par Kant (1785)}

Si Herder éprouve une réelle difficulté à offrir autre chose que des programmes, la recension de Kant marque une nouvelle station dans le rapport de cette difficulté. Or que s'est-il passé en 1784? Parce que le contexte de la polémique est bien documenté ${ }^{30}$, nous serons assez brefs. Lors de la parution des ldées pour une philosophie de l'histoire de l'humanité, qui ne seront jamais terminées, notons-le, Schütz 
demande à Kant d'écrire un compte rendu du livre anonyme pour l'Allgemeine Literaturzeitung. Sauf qu'à ce moment précis, Kant, qui vient d'offrir Réponse à la question: Qu'est-ce que les Lumières, sent l'avènement d'une époque nouvelle, l'Aufklärung, grâce à laquelle les hommes «sortiront hors de l'état de minorité dont ils sont eux-mêmes responsables". Assuré d'être le chef de file de ce mouvement et retrouvant dans les Ideen un auteur qui aime les effets et qui joue au génie ${ }^{31}$, Kant n'est pas dans l'état d'esprit pour le ménager : il l'attaque dans son écriture, son style et sa méthode. Ce qui irrite surtout l'auteur de la Critique de la raison pure, c'est la méthode comparative ainsi que le style de Herder, car ce dernier fait un usage généralisé des métaphores et des analogies ${ }^{32}$. À l'instant même où il prend connaissance du compte rendu, Herder a de nombreuses raisons de s'inquiéter de l'incompréhension dont il est victime. Tout se passe comme si, en effet, il était l'objet d'une exclusion du champ légitime de la philosophie dont il n'avait pas, sur le coup, soupçonné toute la gravité. Que fera Herder devant les accusations du professeur Kant? Réponse : il se tournera vers l'autre géant, Hamann, qu'il voudra voir à ses côtés, au nom de l'amitié, contre Kant. À titre d'ami, il lui écrit :

Car à quel point il est perfide et pubertaire, pour tirer à partir de sa préface le plan d'un livre inachevé et à peine commencé pour y installer une idée de la sorte et faire comme s'il n'existait aucun livre de ce genre au monde (...) pour se mettre aussitôt à la tâche d'assassiner un livre incomplet de la façon qui lui est le plus contraire (... $)^{33}$

On notera qu'au cœur de cette polémique, qui porte sur plusieurs aspects et qui durera plusieurs années, se joue encore une conception du livre de Herder. En effet, les Ideen était bien un livre, mais un livre en construction, un work in progress, un nouveau programme, pour un public allemand encore à venir. Dans la mettre lettre, nous lisons:

Je me demandais qui, ici où là en Allemagne, pouvait écrire en se plaçant si totalement à l'extérieur de l'horizon de l'Allemagne et de mon livre jusqu'à ce qu'on chuchote à l'oreille et que l'on dise bien haut : c'est le grand métaphysicien Kant, à Köningsberg, en Prusse ${ }^{34}$.

Or le livre recensé par Kant n'est même pas terminé. En fait, la première parution comprend 5 des 20 livres prévus! En vérité, Kant 
critique un texte virtuel, un ouvrage à venir. Pour comprendre la fragilité de Herder, il faut se demander ce qu'aurait dit Kant, en revanche, si Herder avait rédigé une recension décapante de la première partie de la Critique de la raison pure en la faisant passer pour le système kantien? La recension de Kant est, bien entendu, intempestive envers le projet de Herder. Celui-ci est d'autant plus déçu qu'il souhaitait voir son «livre» lu par les jeunes générations. Pour donner enfin une idée de l'impact de Kant, rappelons que Herder revient sur la recension dans une lettre à Knebel, en mars, et lui écrit : "Je n'ai presque plus de plaisir à écrire» 35 .

On peut ainsi expliquer le refus de toute critique chez Herder par le refus de toute signature. La querelle de 1785 s'explique d'autant mieux lorsque l'on comprend que Herder considère tous ses "livres" comme inachevés, comme étant sans auteur. II se croit ainsi à l'abri de toute critique, y compris celle de l'auteur de la Critique de la raison pure, comme il l'écrit à Hamann, pensant par là ôter toute légitimité aux critiques de ses livres qui, en vérité, n'en sont pas ${ }^{36}$. Ce ne sont que des programmes, des projets. Devant ce chantier, pensera Herder, Kant, le professeur, n'est pas plus apte que quiconque à porter un jugement sur ce que sont des «Idées» pour l'histoire.

II importe de souligner ici comment Herder interprète le vocabulaire paternaliste que Kant, ce loyal sujet de la monarchie absolue, utilise dans sa recension, la recension d'un programme qui ne veut qu'ouvrir enfin la perspective historique aux "peuples»37. Comment Herder peut-il se sentir face à cette figure du père qui le renie, «mein Lehrer", ou à cette figure de l'autorité universitaire, "le maître es sept art" (sic), le chassant hors du champ philosophique. Que dire de Kant qui considère encore et toujours Herder comme son étudiant, lui qui a plus de 40 ans? Le pasteur se sait imparfait, comme l'expliquent les lignes de Auch eine Philosophie où il refuse de juger les hommes, n'étant qu'un point dans le Grand livre de Dieu. Aussi fidèle à Hamann et à sa conception spirituelle du livre vue plus haut, Herder rappelle à Hamann que Kant n'est pas fidèle à «l'esprit» du livre. La recension de M. Kant (Herr Kant) est donc marquante et doit s'interpréter dans le sillage d'une vision luthérienne, assurément hamannienne, du livre, Herder étant toujours incapable d'écrire comme il le voudrait. Une autre analyse permet de mieux cerner un enjeu somme tout majeur de cette polémique, à savoir la divergence d'opinion de nos deux auteurs sur ce que doivent être les Lumières. 
Une comparaison des conceptions de l'Aufklärung de Kant et de Herder est utile ici, car elle permet de comprendre, dans un contexte plus large il est vrai, les textes des deux auteurs ainsi que le public qu'ils visent par leurs écrits. Si Kant veut depuis toujours réformer philosophiquement l'entendement, Herder s'appuie depuis 1767 sur l'étude du langage et de l'histoire. Si Kant distingue, dans Projet de paix perpétuelle et Réponse à la question : Qu'est-ce que les Lumières?, l'usage privé et l'usage public de la raison, Herder continue de défendre les expressions multiples et incommensurables des cultures. Tandis que Kant propose un principe abstrait de publicité (Öffentlichkeit) comme méthode et balise de l'Aufklärung, doublée d'une conception assurément autoritaire, paternaliste et monopolistique de la raison, avec lui purifiée, Herder conçoit plutôt les Lumières de manière "organique" comme un public fondé dans l'expérience ordinaire du langage et la voix du peuple. Pour Herder, les professeurs sont des citoyens et doivent, dans l'espace public, travailler avec la jeunesse. Si l'éducateur Kant est persuadé qu'il faut apprendre à faire un usage public de la raison et que le mot d'ordre de l'Öffentlichkeit appelle un idéal cosmopolitiste, Herder n'en demeure pas moins convaincu que le progrès ne passe pas exclusivement par la raison, mais qu'il est possible par les forces à l'œuvre dans le langage, surtout poétique, c'est-à-dire l'histoire. II ne revient donc pas au monde cultivé seulement de créer un public, à la manière française, mais au peuple de se constituer lui-même en public. Face à Kant qui cherche par tous les moyens à se situer à l'extérieur d'où s'écrit la philosophie, Herder rédige de l'intérieur «l'histoire culturelle du genre humain" (Kulturgeschichte der Menschheit). Nous reviendrons sur ce parallèle fort instructif un peu plus loin. Pour l'instant, tirons les leçons de la polémique avec Kant sur l'histoire du genre humain pour ensuite tourner notre regard vers la période de maturité, durant laquelle les choses ne s'arrangent guère pour Herder.

La recension des Ideen nous permet finalement de relever à nouveau la difficulté herméneutique que pose l'œuvre de Herder. Car la polémique ne fait pas qu'illustrer les difficultés d'écrire sur l'histoire chez Herder, elle illustre aussi combien différemment les deux philosophes conçoivent la tâche et le rôle de l'écrit. Or si la cible s'est déplacée entre 1774 et 1785 , les tergiversations dans l'écriture de Herder sont demeurées : comme dans Une autre philosophie de l'histoire, les Ideen affirment et nient tout à la fois le progrès du genre humain. Dans les deux ouvrages, Herder continue de considérer la 
Bible comme le premier livre, le premier récit de l'évolution du genre humain et condamne la finalité providentielle comprise comme finalité divine. Mais pour saisir les difficultés de Herder, il n'est pas inutile de comprendre aussi à quel public il s'adresse. Faute d'espace, nous serons peu bavard au sujet de la période de Weimar.

\section{Notes sur la période de Weimar (1776-1803)}

À Weimar, Herder sait que la ville n'en a que pour le classicisme. N'est-ce pas d'abord la ville de Goethe et de son administration? On ne le dira peut-être jamais assez, mais Herder, qui ne s'entend pas très bien avec Schiller, doit composer avec cette réalité. II n'est dès lors pas surprenant que durant cette longue période, il continue à entretenir des rapports forts particuliers avec ses écrits, ce que l'on doit résumer ici.

En effet Caroline, sa femme, doit l'aider avec son œuvre : c'est elle qui prend en charge les liens avec les éditeurs, qui s'occupe de la correspondance, de l'envoi et de la correction des manuscrits et qui gère les rééditions. Dans ce contexte d'insuffisance, Herder peut avouer «ne plus supporter ses livres; sitôt qu'il les voit imprimés (...) ils ont perdu la familiarité qu'ils avaient en manuscrit38». Cet extrait de la correspondance est intéressant parce qu'il prouve encore, s'il en est besoin, à quel point Herder n'a pas appris de la distance séparant le manuscrit du livre publié. Tout se passe encore comme si sa femme Caroline l'aidait à accoucher de ses écrits, l'aidait à les rendre publics, loin de lui, en le maintenant dans l'effectivité de l'écrivain.

Or nous ne comprendrons bien le sentiment de Herder que si nous nous rappelons qu'à Weimar, l'essentiel du travail de Herder consiste à reprendre ses écrits antérieurs. Car si les années de travail ont hypothéqué la santé de l'homme et que la querelle avec Kant n'est toujours pas digérée (Herder fera paraître en 1799 une volumineuse Metakritik de la "Critique de la raison pure" dont le titre est inspiré d'un court texte de Hamann ${ }^{39}$, rédigée en quelques semaines seulement), elles n'ont pas encore apporté la gloire. Ici encore, notre interprétation, qui veut que Herder n'arrive pas à écrire des livres, semble se confirmer : tous ses écrits, y compris ceux de Weimar, se développent en se reprenant sans cesse, visent moins à créer une bibliographie qu'à penser le monde de l'intérieur, du point de vue de l'histoire en action. Herder a bien entendu conscience à Weimar de n'avoir point offert au grand public des monographies, des livres, mais seulement les segments d'une œuvre en mouvement, à venir, les 
livres se donnant à penser dans un horizon mobile. II est difficile pour lui de ne pas y voir un échec. Mais y a-t-il lieu d'y déceler un échec? Un échec présuppose naturellement une ambition, une tentative. La question du rapport entre Herder et sa production, on le constate sans peine, doit s'éclaircir au moyen d'une réflexion sur le rapport de Herder à son public. Le public de Herder est-il en Allemagne, demanderons-nous, en guise de conclusion?

\section{À quel public s'adressait Herder?}

En vérité, Herder s'est toujours lui-même posé la question. À 21 ans, il proposait une conception "ouverte» de ce que devrait être une époque des Lumières (une vision organique selon W. Redekop) dans son texte Avons-nous encore la patrie et le public des anciens? (1766) ${ }^{40}$. De fait, il n'est pas ardu de retrouver au fil de l'œuvre entière cette intuition qu'il manque de vie et de cohésion dans la sphère intellectuelle allemande. Dans sa compréhension du concept de «peuple» (Volk), par exemple, Herder inclut toutes les couches sociales (y compris les femmes) et cherche à favoriser l'interaction entre elles. Voilà peut-être pourquoi Herder, qui professa presque toute sa vie durant des sermons aux gens ordinaires, ne cesse de se concevoir lui-même comme un "écrivain populaire" (Volksschriftsteller), dont la rhétorique, moins philosophique que poétique, tournée vers le public qui doit se rassembler sous le nom de peuple, vise à promouvoir la vie. Cet usage positif de la rhétorique, qui n'a pas échappé à Gadamer ${ }^{41}$ et dont Kant fait le plus grand vice de sa philosophie de l'histoire, illustre très bien selon nous l'objectif qu'il poursuit : rendre fluide, vivant et accessible à tous le dialogue dans l'Aufklärung. C'est pourquoi Herder, l'ancien étudiant de médecine, utilise à répétition les analogies organicistes, les images du développement de toutes les espèces de la terre. Par celles-ci, il marque son opposition à la pensée mécaniste, fille de Descartes, toujours très à la mode au XVIIIe siècle, afin de lui substituer la force, la vie et l'horizon.

Au sujet de la recherche d'un public herdérien, la lecture historique qu'effectue Herder durant toutes ses années de production doit être mise à contribution. En effet, très tôt Herder avait relevé que la formation des peuples passait par leur capacité à se donner une langue et une poésie, à se créer un monde à partir de leur langage. La Grèce et la Rome antiques n'avaient-elles pas, suivant son essai de 1778 Über dir Würkung der Dichtkunst auf die Sitten der Völker in alten und neuen Zeiten (De l'effet de la poésie sur les mœurs des 
peuples dans les temps anciens et nouveaux), de grands poètes et de véritables forums où s'exprimaient les citoyens? Dans une époque où apparaît la culture de l'imprimé, où le livre tend à se généraliser, Herder est convaincu qu'il y a une réelle différence entre entendre et lire. D'après sa comparaison entre l'antiquité et la modernité, il manque au texte écrit un pouvoir, un effet (Würkung). Que veut dire Herder ici?

C'est que l'expression poétique reste lumière et passion : don divin, elle engendre l'action morale et le caractère. Toujours selon Herder, le langage poétique a un effet bien réel sur les personnes et il conduit à la formation d'une humanité. L'espace politique ne s'est jamais affranchi de la littérature et de la poésie, estime-t-il. Ce n'est donc pas un hasard si, face aux Encyclopédistes, Herder s'investit de la mission de faire "sonner» les mots, de redonner droit de cité à la métaphore, afin d'éveiller ses lecteurs au devenir. II ne s'agit ni plus ni moins que de faire passer le texte de lettre morte à action, en utilisant la poésie qui transporte et accomplit le genre humain.

Le véritable public des Lumières, qui aura retenu les leçons de l'histoire, enrichira selon lui le dialogue entre les arts et des sciences, entre les sentiments et la raison, tout en reconnaissant une grande place au premier livre, la Bible, qui est l'enseignement vivant de Dieu aux hommes. Dans les Lettres sur l'étude de la théologie de 1781, Herder ne pense pas que la nature est Dieu, mais continue de reconnaître en la Bible le texte vivant, celui qui peut former des hommes responsables. Si la Bible demeure si importante à ses yeux, c'est bien parce que son écriture est vivante et qu'elle exige une appropriation de sa signification. Herder est certainement celui qui a développé la philosophie de l'histoire la plus respectueuse des peuples, celle qui s'est montrée la plus consciente des forces et des différences, lorsqu'il a fait paraître Auch eine Philosophie, en 1774, le premier et peut-être le seul ouvrage capable de comprendre le progrès historique avec l'universalité de la Parole depuis les Méditations bibliques de Hamann.

Au tournant des années 1800, Weimar n'est pas encore la capitale culturelle de l'Allemagne que souhaite Herder. En effet, si la majeure partie de l'action se passe à léna et Berlin, l'Allemagne que veut l'écrivain reste à venir, toujours en projet, en formation. Influencé par Hamann, on l'a dit, mais aussi par Lessing qui ne cherchait qu'à mettre sur pied un théâtre national allemand, Herder répétera très 
souvent, en cela d'accord avec le jugement de Madame de Staël, que les Allemands n'ont pas de peuple, pas de nation, pas de public, pas de langue ni de poésie ${ }^{42}$. Que doit-on comprendre de cet amer constat?

Pour le savoir, il convient de terminer notre parallèle entre Kant et Herder, débuté plus haut et de préciser la vision des Lumières que Herder voudrait bien voir émerger en Allemagne. L'espace public de 1785 n'est pas le Publikum au sens où l'entend Herder. Ne croyant pas au système, il propose un "art de parler" philosophique qui s'adresse au peuple revendiquant une identité culturelle. La réponse critique de Kant à la question de l'Aufklärung ne peut satisfaire Herder : Kant veut éduquer le public, mais il écrit pour un public artificiel, soumis aux canons de la pureté, intellectuel et éloigné de la force qui fait l'histoire. Le vrai Publikum est porté par une langue nationale, une langue maternelle. La réception littéraire et philosophique à Weimar constitue pour lui une pâle copie des idéaux dépassés de la culture française. Herder est donc d'avis, avec Nicolaï à Berlin, que les universitaires doivent sortir de leur secte pour rejoindre les vingt millions d'allemands qui attendent de les lire, un peuple qui développe déjà un nouveau sens de la culture et de l'identité depuis la guerre de Sept ans (1756-1763). Le public de Herder, tel que décrit ici dans la confrontation avec Kant, n'aura évidemment pas été au rendez-vous.

Nous espérons avoir montré que l'incapacité de Herder à écrire un livre tient aussi bien à son rôle de trait d'union entre Hamann et Kant qu'à sa situation de voyageur et de pasteur. Quant à sa philosophie de l'histoire, qui culmine dans la polémique avec Kant, elle reste tributaire de sa vision du livre, de l'écriture et des Lumières. Consciente qu'elle est des forces historiques et de la transmission du sens dans le langage, sa lecture s'avère toujours stimulante. S'appuyant sur le sens commun et des "effets" rhétoriques, les textes de Herder nous donnent à penser cette grande vérité (si grande que Gadamer s'en est inspiré pour construire une philosophie herméneutique) que notre langage porte toujours en lui une conception du monde et une histoire. Si les textes de Herder sont difficiles à lire, en revanche ils s'éclairent les uns avec les autres à la faveur d'un dialogue se reprenant sans cesse. Herder aura sans doute produit une œuvre "ouverte» parce qu'il n'a jamais visé l'achèvement définitif ou un public assuré. II aura écrit sans connaître les habitudes et les mœurs de son public. Ce qui n'est peut-être pas si grave, enfin. Car le 
pasteur, pour qui la poésie constitue la vérité et la vie du langage, aura écrit des livres inachevés parce qu'inachevables. Mais en ce sens, les difficultés de Herder à écrire un livre et à rejoindre un public n'indiquent peut-être pas un échec. Au contraire, ces difficultés, gravées dans sa philosophie de l'histoire, peuvent nous apprendre beaucoup, à commencer par cette évidence que les livres, qu'ils soient achevés ou inachevés, qu'ils soient primés ou passés sous le manteau, portent en eux la promesse d'un avenir meilleur et, par conséquent, ne meurent jamais avec leurs auteurs. Le lecteur attentif aux difficultés de Herder retiendra sans doute que, si le public n'est jamais assuré et que le langage travaille toujours notre histoire, c'est le chemin qui mène au livre qu'il faut, plus que jamais, chercher à penser.

\section{Dominic Desroches Université de Montréal Département de philosophie desrochd@magellan.umontreal.ca}

1. Germaine de Staël, De l'Allemagne, Paris, G-F, 1966, t. II, p. 65.

2. Comme le veut le sous-titre du livre : für die lange Weile des Publicums zusammengetragen von einem Liebhaber der langen Weile. Mit einer doppelten Zuschrift an Niemand und an Zween. Et la dédicace à la page suivante : «An der Publicum oder Niemand, den Kunbaren". Sokratische Denkwürdigkeiten, Reclam, Stuttgart, 1993. Dans le même sens, Hamann ne disait-il pas dans la Lettre volante qu'il "prêchait dans le désert"?

3. J.G. Hamann, Werke, Ed. Roth, Berlin, 1821, ici Band I, 509. P. Klossowski a traduit cette lettre très importante dans Le Mage du Nord, Fata Morgana, 1988, p. 44-52.

4. J.G. Hamann, Werke, I. 509. Nous suivons ici la traduction de P. Klossowski (voir note précédente).

5. Sur le principe de la coïncidence des opposés, essentiel à la pensée de Hamann, on se référera également à une autre lettre à Jacobi, celle du 16 janvier 1784 .

6. J.G. Hamann, Werke, I, p. 99.

7. Voir l'introduction de Max Rouché à Auch eine Philosophie (Une autre philosophie de l'histoire), Paris, Aubier, p. 76-85.

8. Cf. B. Redekop, Enlightenment and Community : Lessing, Abbt and Herder and the quest for a German Public, Mc Gill-Queens University, 2000, p. 30-34.

9. Kant invite Herder à développer une œuvre dans la lignée de Montaigne, Hume et Pope I Voir la lettre de Kant à Herder du 9 mai 1768. Pour la correspondance, Herders Briefe, Volksverlag, Weimar, 1959. Au sujet de ce développement, lire P. Pénisson, J.G. Herder, La raison dans les peuples, Paris, Cerf, p. 57-60.

10. J. Habermas, L'espace public. Archéologie de la Publicité comme dimension constitutive de la société bourgeoise, Paris, Payot, 1978, p. 114. 
11. H.-G. Gadamer, Nachwort à Auch eine Philosophie der Geschichte zur Bildung der Menschheit, Theorie 1, Suhrkamp, 1967, 148-149. La lecture de Gadamer mérite un détour parce qu'elle recentre pertinemment le livre "polémique" de Herder en son siècle, c'est-à-dire aux originęs d'une conscience historique, en même temps qu'elle le situe toujours face aux courants luthérien et romantique.

12. H.-G. Gadamer, op.cit, p. 150.

13. Auch eine Philosophie, Theorie 1, Suhrkamp, 1967, 103; trad. fr. Max Rouché, Paris, Aubier, 1964, p. 301

14. Ibid.

15. Auch eine philosophie, 103-104; trad. fr., p. 302-303,

16. Auch eine philosophie,106; trad. fr., p. 307.

17. Ibid.

18. Ibid.

19. Auch eine Philosophie,137; trad. fr., p. 367.

20. À l'aide d'une métaphore digne de Hamann, il écrit dans Auch eine Philosophie : "Chaque nation porte en elle son ęentre de félicité de même que chaque sphère a en elle son centre de gravité", op. eit., p. $1 \varepsilon \mid 3$.

21. Après avoir donné une conférenẹe en français dans un camp de prisonniers, il publie en 1942 Volk und Geschichte im Denken Herders (Peuple et histoire dans la pensée de Herder) dans lequel il s'intéresse notamment au concept de force dans la pensée de l'histoire.

22. H.-G. Gadamer, «Herder als wegbereiter dir "historischen Bewusstseins", Geist der Zeit, Wesen und Gestalt der Vëlkerw, tradıit dans los cahiers de l'Institut allemand, 2, Paris, 1941 sous le titre : Herder et ses théories de l'histoire. Ici, l'interprétation de P. Pénisson est particulièrement éclairiante.

23. P. Pénisson, op. cit., 93.

24. Voir le Journal de voyage de 1769. Pour un eommentaire précis, P. Pénisson, Johann Gottfried Herder, La raison dans lies peuples, p. 95.

25. Voir Warheit und Methode (WM), Gesammelte Werke, B. 1, J.C.B Mohr, Tübingen, 1960/1990, p. 307. trad. fr. Vérité et Méthode (VM), trad. Fruchon, Grondin, Merlio, Paris, Seuil, 1996, p. 324

26. Ibid.

27. P. Pénisson, J.G. Herder. La raison dans les peuples, p. 83

28. P. Pénisson, J.G. Herder; La a kaison dans les peuples, p. 20.

29. Ibid. C'est à Gadamer que revient le rnérite d'avair développé cette idée. Dans Vérité et Méthode, il écrivait, contre la tentation de s'en remettre à l'immédiateté : "Quand nous cherchons à comprendre un phénomène historique à la distance quil déternine glabalement notre situation, herménutique, nous sommes toujours soumis aux effets (Wirkungen) de l'hist:oire de l'action (Wirkunggeschichte)". WV, $306, V M$, p. 322.

30. À propos de la querelle, on lira P: Pénission, «Kant et Herder : le recul d'effroi de la raison", in Revue germanique internationale, numéro 2, 1996, p. 63-74. M. Crépen, "Le jeu de Kant et de Spinoza dans le liv re XV des Idées sur la philosophie de l'histoire de l'humanité", in Les Elutes philosiophiques, Numéro 3, 1998, p. 389-402. A. Philolenko, "Kant et Herder - une polémique", in La théorie kantienne de 
l'histoire, Paris, Vrin, 1986, p. 125-145. M. Rouché, "La polémique contre Kant", in La philosophie de l'histoire de Herder, Thèse, Université de Strasbourg, 1940, p. 310-318.

31. Cf. E. Kant, Compte-rendu de l'ouvrage de Herder : "/dées pour une philosophie de l'histoire de l'humanité»,p. 91-92. Kant écrit : "(...) il maintient toujours dans un vaporeux éloignement, grâce à un jeu de sentiments et d'impressions qui se manifestent comme les effets d'une densité de penséen.

32. II y a tout lieu ici de retrouver un motif décisif de la controverse avec Kant. La méthode comparative et l'usage de l'analogie par Herder sont inacceptables pour Kant. Pour sa part, Kant avait fait un usage plus rigoureux de l'analogie dans sa première critique. Pour Kant, les "analogies de l'expérience" sont des règles selon lesquelles l'unité de l'expérience doit reposer sur une unité nécessaire des perceptions. Pour plus d'informations à ce sujet, voir les articles et ouvrages consacrés à la polémique et cités plus haut.

33. Lettre de Herder à Hamann du 14 février 1785, traduite par P. Pénisson, Herder, la raison dans les peuples, p. 109-110.

34. Ibid.

35. Lettre de Herder à Knebel, 2 mars 1785 : "An meinen Ideen wird schrecklich langsam gedruckt, weil papier fehlest (...) Die Lust zum Schreiben ist mir ziemlich vergangen..."

36. P. Pénisson, «Kant et Herder : Le recul d'effroi de la raison», in Revue germanique internationale, numéro 2, 1996, p. 6.5.

37. Comme W. Redekop le remarque fort pertinemment, à cette époque la société allemande est encore très paternaliste' (paternalistic) et est basée sur la notion de hiérarchie naturelle, d'aristocratie; On y trouve ainsi un respect pour la noblesse et l'autorité patriarcale. op. cit., p. 30. SSur la monarchie absolue, lire les travaux de Vierhaus, trad. ang. Germany in the Age of Absolutism, p. 50-52.

38. Herder Briefe, Lettre du 7 octobre 17910 à J.G. Müller.

39. J.-G. Hamann, “Métacritique sur le purisme de la raison" (1784) est la première recension de la célèbre Critique de Kant. Sa rédaction, on parle des ébauches, fut commencée dès la lecture des épreuves du livre de Kant obtenu par Hamann chez l'imprimeur Hartknoch, en juillet 1781. Le texte est resté inédit jusqu'en 1801 et, du vivant de Kant, seuls Herder et Jac:obi en avaient pris connaissance. Herder, lorsque vient le temps de mieux faire porter ses coups contre Kant, reprend une partie de l'argumentaire de Hamann, au point même d'y emprunter le titre... Pour les curieux, le texte a été traduit en français par $\mathrm{H}$. Corbin, Hamann et le luthérianisme, Berg, 1985, p. 139-147 et par J. Colette, à qui l'on doit les Écrits de Hamann de Hegel, dans Philosophie, 1997, p. 3-13.

40. Pour ce développement, on lira avec profit W. Redekop, op. cit., p. 168-220.

41. H.-G. Gadamer, "Herméneutique classsique et philosophie», in La philosophie herméneutique, trad. J. Grondin, 110. Gadamer remarque que, malgré les efforts de Herder, la rhétorique perd de son influence aux $X I X^{e}$ et $X X^{e}$ siècles.

42. Ce constat était aussi partagé par Wieland. Cf. W. Redekop. op.cit, p. 54. 